\title{
Background incidence rates of adverse pregnancy outcomes in the Netherlands; data of 2006-2018.
}

\author{
Maarten Immink ${ }^{1}$, Sanne Koole ${ }^{2}$, Mireille Bekker ${ }^{3}$, Floris Groenendaal ${ }^{3}$, Jeanet \\ Kemmeren $^{1}$, Hester de Melker ${ }^{1}$, and Nicoline van der Maas ${ }^{1}$ \\ ${ }^{1}$ National Institute for Public Health and the Environment \\ ${ }^{2}$ The Dutch Perinatal Registry, Perined \\ ${ }^{3}$ Wilhelmina Children's Hospital, University Medical Center Utrecht, and Utrecht \\ University
}

July 24, 2020

\begin{abstract}
Objective To assess background rates of adverse pregnancy outcomes before implementation of a maternal pertussis immunisation programme in the Netherlands, to put into perspective the safety concerns about such outcomes following immunisation. Design Retrospective cross-sectional study. Setting and population Dutch pregnant women and their infants who were registered in the Dutch Perinatal Registry in the 2006-2018 period. Births of [?]500g birth weight and [?]24+0w gestational age were included. Methods Annual numbers of adverse outcomes were used to calculate incidence rates per 10,000. Trends with movingaverage-lines over the past 3 years were plotted, with $95 \%$ confidence interval. Main Outcome Measures Adverse outcomes at maternal and neonatal level. Results From 2006 through 2018, yearly numbers of pregnancies ranged between 158,868-175,710. Numbers of newborns ranged between 161,307-178,874, of whom 160,838-178,177 were live-born. Most outcomes were stable over time. Between 2006-2011, occurrence of labour induction increased by $68 \%$, and postpartum hemorrhage increased by $25 \%$. Both stabilised from 2011 onwards. Perinatal mortality up to day 7 or 28 postpartum decreased by $38 \%$ and $37 \%$, respectively. Occurrence of low Apgar score among preterm infants born before 37+0w gestational age and among term infants increased by $19 \%$ and $27 \%$, respectively. Conclusions Our study on background incidences showed notable increases over time in occurrence of labour induction, postpartum hemorrhage and low Apgar score, while showing a considerable decrease in overall perinatal mortality. These findings should be considered when interpreting data on adverse events occurring since Tdap vaccination was implemented.
\end{abstract}

\section{Introduction}

Infants too young to be vaccinated depend on maternal antibodies for protection against infectious diseases, such as pertussis. These infants are at increased risk of severe pertussis complications, leading to hospitalisation and sometimes even death. (1-4) During pregnancy, IgG-antibodies, produced in the mother after infection or immunisation, then transferred to the fetus through the placenta. Recent studies show that an early-second-trimester tetanus, diphtheria and acellular pertussis (Tdap) vaccination significantly increases pertussis-specific antibodies in term and preterm infants compared to third-trimester vaccination. $(5,6)$ Therefore, an increasing number of countries now recommend Tdap immunisation earlier in gestation. In the Netherlands since December 2019, the National Immunisation Programme (NIP) has offered Tdap vaccination to all pregnant women of at least 22 weeks (w) gestational age (GA).

Studies in (non-pregnant) women have shown that injection site reactions and systemic reactions like headache and fatigue were related to Tdap vaccination. (7-9) Its use during pregnancy has been extensively studied and is considered safe, without increased risk of a wide range of adverse pregnancy outcomes. 
(10-13) Some non-randomised studies have reported a small increased risk of chorioamnionitis in women who were immunised against pertussis, but found no increased frequency for clinically relevant sequelae, e.g. earlier onset of labour resulting in preterm delivery. (11, 14-16)

Various adverse pregnancy outcomes such as (pre-)eclampsia, preterm delivery, or low birth weight emerging shortly after Tdap immunisation could be considered an adverse reaction to the vaccine. Therefore, despite the favorable safety profile of the Tdap vaccine, implementation of such maternal immunisation programmes can raise public safety concerns. To distinguish legitimate safety concerns from coincidental events following immunisation, we assessed background rates of adverse pregnancy outcomes at maternal and neonatal level prior to the 2019 implementation of a maternal Tdap immunisation programme in the Netherlands. Assessing these background rates is important for evaluating the safety of the Tdap vaccination since its implementation and also for evaluating future maternal immunisation strategies.

\section{Methods}

\section{Data source}

We used routinely collected data from the Dutch Perinatal Registry (DPR; www.perined.nl) to extract information about adverse pregnancy outcomes from 2006 through 2018. The DPR database contains the anonymous and interconnected data of national registries of four professional organisations that provide perinatal care in the Netherlands, i.e. midwives, general practitioners, gynecologists, and pediatricians. It contains prospectively obtained population-based data on pregnancies and the care provided for newborns, e.g. interventions, referrals, deliveries and admissions, of approximately $98 \%$ of all deliveries in The Netherlands. (17)

\section{Study population and setting}

Our study population consisted of $98 \%$ of all pregnant women and their infants recorded by DPR from 2006 through 2018. Births of [?]500 g birth weight and [?]24+0 w GA were included. All data obtained from the DPR database were extracted from individual medical records and rendered anonymous, taking the European privacy policy into account. Therefore, medical ethical approval and individual informed consent for participation were not necessary. (18)

\section{Outcome measures}

Box 1 shows the outcomes we selected for this study. Case definitions are described in the supplement. Variables requiring an additional explanation are described below.

Box 1. Adverse pregnancy outcomes to be possibly assigned to the Tdap vaccination

Maternal outcomes maternal mortality; placental abruption; hypertension; (pre-)eclampsia; rupture of membranes [?]24h

\section{Addidtional explanation of outcomes}

Pre-eclampsia and eclampsia were combined because separate data were not available. Postpartum hemorrhage was defined as [?]1000 $\mathrm{ml}$ blood loss in the first 24 hours (h) after delivery.

Since we only included cases of [?] $24+0 \mathrm{w}$ GA, stillbirth was defined as death in the period between $24+0 \mathrm{w}$ GA and birth. Likewise, neonatal mortality was defined as death in the period from birth until 28 days (d) after birth. Perinatal mortality was calculated for infants until $7 \mathrm{~d}$ or $28 \mathrm{~d}$ after birth.

SGA and LGA were determined using Hoftiezer charts, in which percentiles of birth weight versus GA have been calculated with SGA and LGA defined as $<10^{\text {th }}$ percentile and $>90^{\text {th }}$ percentile, respectively. (19)

We decided to restrict neonatal hospital admissions to infants who were hospitalised due to a medical indication. Infants born after a caesarean section usually remain hospitalised for a short time, but this reflects standard care and is not in itself an adverse outcome. 
Idiopathic respiratory distress syndrome (IRDS) was determined only for preterms, because its prevalence in term infants is extremely low.

Mean length of hospital stay cannot be used as an adverse pregnancy outcome, because it is directly affected by GA. Therefore, the mean difference in days between hospital discharge date and the expected delivery date is used as a proxy for the need of hospitalisation in preterms with divergent lengths of GA.

Outcome measures directly associated with GA, e.g. low Apgar score at $5 \mathrm{~min}$, were (also) analyzed in categories of prematurity; extreme preterms $(<28+0 \mathrm{w} \mathrm{GA})$, all preterms $(<37+0 \mathrm{w} \mathrm{GA})$ and infants born at term ([?] 37+0w GA).

Because in the Netherlands the maternal Tdap vaccination can be administered from $22+0 \mathrm{w}$ GA onwards, we additionally specified incidence rates (IR) of stillbirth, neonatal mortality and perinatal mortality for all cases between 22+0w GA and 23+6w GA.

\section{Statistical analysis}

To calculate IRs per 10,000 from 2006 through 2018, we used annual numbers of pregnancy outcomes, divided by the total number of pregnancies or births each year for maternal and infant outcomes, respectively. Additional moving averages of the past 3 years were plotted from 2008 onwards, containing a $95 \%$ confidence interval (CI). Data retrieval was performed using R-software version 1.2.5042.

\section{Results}

From 2006 through 2018, yearly numbers of pregnancies ranged between 158,868 and 175,710. Yearly numbers of newborns ranged between 161,307 and 178,874 , of whom 160,838 to 178,177 were live-born. Table 1 shows IRs of included maternal and infant outcomes per 10,000 from 2006 through 2018.

Figures 1 and 2 show time trends for all maternal and neonatal outcomes.

IRs of labour induction increased by $68 \%$ between 2006 and 2011 (1228 to 2067 per 10,000) (Figure 1). Afterwards, the IR stabilised, ranging between 2022-2148 per 10,000. Sub-analyses showed that the mean GA between 2006 and 2011 decreased by three days among women who gave birth after labour induction at [?] 40+0w GA (from 41+4w GA in 2006 to $41+1 \mathrm{w}$ GA in 2011). IRs of postpartum hemorrhage increased by $25 \%$ (504 to 631 per 10,000) in the same period and stabilised from 2011 through 2018, ranging between 611-646 per 10,000. Over all, the IRs of artificial labour remained stable over the observed period, with caesarian section fluctuating between 1470 and 1507 per 10,000, whereas vacuum extraction and extraction by forceps decreased, the former from 980 to 695 per 10,000 and the latter from 55 to 12 per 10,000.

IRs of LGA fluctuated, with a maximum of 1114 per 10,000 in 2008 and a minimum of 968 per 10,000 in 2015 (Figure 2). IRs of SGA decreased by $11 \%$ (1176 to 1047 per 10,000) between 2006 and 2018. IRs of stillbirth decreased by $43 \%$ ( 51 to 29 per 10,000). IRs of neonatal mortality decreased by $25 \%$ (28 to 21 per 10,000). As a result, IRs of perinatal mortality decreased by $38 \%$ (73 to 45 per 10,000) up to $7 \mathrm{~d}$ postpartum and $37 \%$ (79 to 50 per 10,000) up to $28 \mathrm{~d}$ postpartum. IRs of severe congenital malformations decreased by $45 \%$ (29 to 16 per 10,000) from 2006 through 2018. IRs for neonatal hospital admissions and NICU admissions showed fluctuations over the observed period, with IRs ranging between 1186-2209 per 10,000 for neonatal hospital admissions and 207-467 per 10,000 for NICU admissions.

The number of births [?]22+0w and [?]23+6w GA ranged by 25 to 30 per 10,000 over the 2006-2018 period. Among these infants, perinatal mortality was nearly $100 \%$ at all times over the observed period; $62-70 \%$ died during pregnancy and 29-36\% died shortly after birth.

IRs of prematurity slightly decreased from 2006 through 2018 in both preterm categories, $<28+0 \mathrm{w}$ GA and $<37+0$ w GA, with a decrease of $7.7 \%$ (39 to 36 per 10,000) for those $<28+0$ w GA compared to $9.4 \%(766$ to 679 per 10,000) for those <37+0w GA. IRs of low Apgar score increased by $19 \%$ (878 to 1044 per 10,000) among preterms $<37+0 \mathrm{w}$ GA compared to $27 \%$ (108 to 137 per 10,000) among among term infants (Table 2). Low Apgar score was most prevalent among preterm infants $<28+0 \mathrm{w}$ GA and fluctuated over the entire 
study period with a range of $4592-5631$ per 10,000. Likewise, IRDS was most prevalent among preterms $<28+0 \mathrm{w}$ GA. Between 2006 and 2007, we saw an increase in IRDS of 69\% (3521 to 5952 per 10,000) in preterms $<28 \mathrm{w}$ GA, which gradually decreased by $25 \%$ between 2007 and 2018 (5952 to 4492 per 10,000). The mean number of days between hospital discharge date and expected delivery date for all preterm infants ranged between 22 and 26 .

\section{Discussion}

The results of the present study show that over 13 years (2006-2018), the occurrence of most pregnancy outcomes remained rather stable in the Netherlands, with no substantial changes over time. However, notable changes included increases in the occurrence of labour induction, postpartum hemorrhage, and low Apgar score, whereas a decrease was observed in overall perinatal mortality.

Changes in IRs of pregnancy outcomes may be partially explained by changes in medical care. Since 2007, the guidelines for post-term pregnancies allowed the possibility of labour induction earlier in gestation: after $41+0 \mathrm{w}$ GA instead of after 42+0w GA. (20) Until 2011, we observed an increase in occurrence of labour induction. In the same period, we observed an increase in occurrence of postpartum hemorrhage. Despite the simultaneously occurring increases, there is no evidence to suggest an association between labour induction and postpartum hemorrhage. $(21,22)$ Since 2011 , both have stabilised, so the most recent IRs reflect the current background incidence and are not expected to increase or decrease in the near future.

The decreased occurrence of stillbirth over the observed period led to a decrease in overall perinatal mortality, i.e. the sum of fetal (stillbirth) and neonatal mortality. The decrease in stillbirths may result in an increased number of infants who are live-born but vulnerable and at higher risk of neonatal mortality. This might partially explain the smaller decrease in neonatal mortality relative to the larger decrease in occurrence of stillbirth. The same mechanism holds for low Apgar score. Fewer fetal deaths, and therefore a higher percentage of more vulnerable infants might have partially led to the small increase in occurrence of low Apgar score over the observed period. The decreased occurrence of severe congenital malformations might be partially explained by the introduction of the 20-week fetal anomaly scan in 2007, possibly resulting into more terminations of pregnancy before $24+0 \mathrm{w}$ GA. (23) For IRDS among preterms $<28 \mathrm{w}$ GA, we observed an increase from 2006 to 2007. In 2006, the Dutch guidelines for active treatment of extremely preterm neonates advised lowering the GA threshold for active intervention from $26+0$ to $25+0 \mathrm{w}$ GA. (24)

Perinatal mortality for infants born between $22+0 \mathrm{w}$ GA and $23+6 \mathrm{w}$ GA was investigated to give context to the adverse events that occurred after maternal Tdap vaccination was administered in the first weeks after the earliest opportunity of a maternal Tdap vaccination within the NIP. We found no substantial changes in IRs over the entire observed period. However, in the 2010 definition of viability was changed to a threshold of at least $24+0 \mathrm{w}$ GA. This might have introduced incompleteness into the DPR data, which could have affected our findings.

Chorioamnionitis is not registered in the DPR. Studies suggesting that Tdap immunisation increases the risk of chorioamnionitis have not reported associations with other maternal or perinatal adverse outcomes. (11, $14,15)$ The clinical relevance of chorioamnionitis is unclear. A diagnosis of chorioamnionitis is solely based on clinical criteria, i.e. fever and two of the following: uterine tenderness or maternal or fetal tachycardia or foul/purulent amniotic fluid. Therefore, clinical chorioamnionitis is diagnosed with variable sensitivity and low specificity. (25) Sequelae to this diagnosis should be more extensively studied in relation to Tdap immunisation.

Two other studies have described adverse outcomes in pregnancy associated with maternal vaccination safety in a national population setting. An observational study in New Zealand reported mean incidences of $4.7 \%$ for preterm delivery (defined as $<37+0 \mathrm{w}$ GA), $8.7 \%$ for postpartum hemorrhage, $7.5 \%$ for rupture of membranes [?] $24 \mathrm{~h}$ pre-labour and $0.4 \%$ for placental abruption (26), which were all similar to our results, i.e. $7.4 \%, 6.1 \%, 6.6 \%$ and $0.2 \%$, respectively. A survey among pregnant active duty United States military women reported results for adverse outcomes that included a mean incidence of $7.7 \%$ for preterm delivery $(<37+0 \mathrm{w}$ GA), which was similar to our finding of $7.4 \%$. (27) 
A major strength of our study is that the DPR provides medical information about $98 \%$ of all pregnancies and births in the Netherlands and is therefore highly representative of the Dutch population. The currently assessed background rates will be used to guide safety surveillance of the maternal pertussis vaccination programme. The data can also be used as information source for strategies to implement other types of maternal immunisation in the future, e.g. maternal vaccination targeting respiratory syncytial virus or group B streptococcus.

Over the years under study, there might have been some changes in the completeness of the registration into DPR. Between 2006 and 2015, we saw an increase in neonatal wards providing data to DPR. This might have had impact on IRs of neonatal hospital admission between those years. Additionally, data of upcoming external neonatal cardiology and neonatal oncology departments in the Netherlands were not incorporated in the DPR database, thereby affecting IRs of these severe congenital malformations.

The advice of the Dutch Health Council to offer a Tdap vaccination to all pregnant women in the Netherlands, was published in 2015. A possible limitation of our study is that in 2018, i.e. before routine maternal vaccination began in December 2019, about $13 \%$ of pregnant women were already Tdap vaccinated at their own expense. (28) It was impossible to distinguish IRs for all outcomes between women who were and were not immunised. However, data for 2018 were in line with data for 2016-2017 for all outcomes, and studies showed no any safety signals. (10-13) Therefore, we do not expect that vaccine coverage had much impact on the IRs discussed in this article.

\section{Conclusion}

Background incidences remain essential when monitoring the safety of an immunisation strategy, especially when a new age- or risk-group is targeted. We found several trends over time for adverse pregnancy outcomes that might be explained by changes in case definitions or standards of care. These data on the incidence of adverse events before the 2019 introduction of Tdap vaccination in the NIP allows more perspective on the incidence of such events since its implementation.

\section{Disclosure of interests}

None of the authors has a conflict of interest to disclose.

\section{Contribution to authorship}

MI, HdM and NvdM designed the study. MI, SK, MB, FG and NvdM contributed to data collection and analysis. MB and FG gave advice on medical explanations to explain changes in IRs over time. MI wrote the manuscript; SK, MB, FG, JK, HdM and NvdM critically revised subsequent versions and approved the final version. MI had full access to all the data in the study and takes responsibility for the integrity of the data and the accuracy of the data analysis.

\section{Details of ethics approval}

Details of ethics approval Medical ethics approval of this study was not necessary because only routinely collected data were used and participants were not imposed a specific deed. The Board of the DPR approved the study. Approval was obtained upon assessment by a privacy commission.

\section{Funding}

The authors received no specific funding for this work.

\section{References}

1. van der Maas NA, van Aerde K, Bont LJ, Bekker MN, Rots N, de Melker HE. [Infection prevention in newborns through maternal vaccination: current insights and developments]. Ned Tijdschr Geneeskd. 2016;160:D411.

2. Vegelin AL, van Vught AJ, Wolfs TF, Kimpen JL, Geelen SP. [Pertussis in young infants]. Ned Tijdschr Geneeskd. 1998;142(49):2657-60. 
3. Amirthalingam G, Andrews N, Campbell H, Ribeiro S, Kara E, Donegan K, et al. Effectiveness of maternal pertussis vaccination in England: an observational study. Lancet. 2014;384(9953):1521-8.

4. Winter K, Nickell S, Powell M, Harriman K. Effectiveness of Prenatal Versus Postpartum Tetanus, Diphtheria, and Acellular Pertussis Vaccination in Preventing Infant Pertussis. Clin Infect Dis. 2017;64(1):38.

5. Eberhardt CS, Blanchard-Rohner G, Lemaitre B, Boukrid M, Combescure C, Othenin-Girard V, et al. Maternal Immunization Earlier in Pregnancy Maximizes Antibody Transfer and Expected Infant Seropositivity Against Pertussis. Clin Infect Dis. 2016;62(7):829-36.

6. Eberhardt CS, Blanchard-Rohner G, Lemaitre B, Combescure C, Othenin-Girard V, Chilin A, et al. Pertussis Antibody Transfer to Preterm Neonates After Second- Versus Third-Trimester Maternal Immunization. Clin Infect Dis. 2017;64(8):1129-32.

7. Munoz FM, Bond NH, Maccato M, Pinell P, Hammill HA, Swamy GK, et al. Safety and immunogenicity of tetanus diphtheria and acellular pertussis (Tdap) immunization during pregnancy in mothers and infants: a randomized clinical trial. JAMA. 2014;311(17):1760-9.

8. Maertens K, Cabore RN, Huygen K, Hens N, Van Damme P, Leuridan E. Pertussis vaccination during pregnancy in Belgium: Results of a prospective controlled cohort study. Vaccine. 2016;34(1):142-50.

9. Fortner KB, Swamy GK, Broder KR, Jimenez-Truque N, Zhu Y, Moro PL, et al. Reactogenicity and immunogenicity of tetanus toxoid, reduced diphtheria toxoid, and acellular pertussis vaccine (Tdap) in pregnant and nonpregnant women. Vaccine. 2018;36(42):6354-60.

10. McMillan M, Clarke M, Parrella A, Fell DB, Amirthalingam G, Marshall HS. Safety of Tetanus, Diphtheria, and Pertussis Vaccination During Pregnancy: A Systematic Review. Obstet Gynecol. 2017;129(3):56073.

11. Kharbanda EO, Vazquez-Benitez G, Lipkind HS, Klein NP, Cheetham TC, Naleway A, et al. Evaluation of the association of maternal pertussis vaccination with obstetric events and birth outcomes. JAMA. 2014;312(18):1897-904.

12. Donegan K, King B, Bryan P. Safety of pertussis vaccination in pregnant women in UK: observational study. BMJ. 2014;349:g4219.

13. Gkentzi D, Katsakiori P, Marangos M, Hsia Y, Amirthalingam G, Heath PT, et al. Maternal vaccination against pertussis: a systematic review of the recent literature. Arch Dis Child Fetal Neonatal Ed. 2017;102(5):F456-F63.

14. Layton JB, Butler AM, Li D, Boggess KA, Weber DJ, McGrath LJ, et al. Prenatal Tdap immunization and risk of maternal and newborn adverse events. Vaccine. 2017;35(33):4072-8.

15. DeSilva M, Vazquez-Benitez G, Nordin JD, Lipkind HS, Klein NP, Cheetham TC, et al. Maternal Tdap vaccination and risk of infant morbidity. Vaccine. 2017;35(29):3655-60.

16. Vygen-Bonnet S, Hellenbrand W, Garbe E, von Kries R, Bogdan C, Heininger U, et al. Safety and effectiveness of acellular pertussis vaccination during pregnancy: a systematic review. BMC Infect Dis. 2020;20(1):136.

17. Perined. The Netherlands Perinatal Registry 2019 [Available from: www.perined.nl.

18. Subjects CCoRIH. CCMO - Legal framework for medical scientific research 2019 [Available from: https://english.ccmo.nl/investigators/legal-framework-for-medical-scientific-research/your-researchis-it-subject-to-the-wmo-or-not.

19. Hoftiezer L, Hukkelhoven CW, Hogeveen M, Straatman HM, van Lingen RA. Defining small-forgestational-age: prescriptive versus descriptive birthweight standards. Eur J Pediatr. 2016;175(8):1047-57. 
20. Gynaecologie NVvOe. Richtlijn Serotiniteit. 2007.

21. Grobman WA, Rice MM, Reddy UM, Tita ATN, Silver RM, Mallett G, et al. Labor Induction versus Expectant Management in Low-Risk Nulliparous Women. N Engl J Med. 2018;379(6):513-23.

22. Miller NR, Cypher RL, Foglia LM, Pates JA, Nielsen PE. Elective Induction of Labor Compared With Expectant Management of Nulliparous Women at 39 Weeks of Gestation: A Randomized Controlled Trial. Obstet Gynecol. 2015;126(6):1258-64.

23. Grevinga M.; Schönbeck YH-M, A.D.; Reijnders, M.E.B.; Detmar, S.B. . Aangeboren afwijkingen in Nederland 2010-2016: Gebaseerd op de Landelijke Perinatale Registraties. 2018.

24. Zegers MJ, Hukkelhoven CW, Uiterwaal CS, Kollee LA, Groenendaal F. Changing Dutch approach and trends in short-term outcome of periviable preterms. Arch Dis Child Fetal Neonatal Ed. 2016;101(5):F391-6.

25. Tita AT, Andrews WW. Diagnosis and management of clinical chorioamnionitis. Clin Perinatol. 2010;37(2):339-54.

26. Griffin JB, Yu L, Watson D, Turner N, Walls T, Howe AS, et al. Pertussis Immunisation in Pregnancy Safety (PIPS) Study: A retrospective cohort study of safety outcomes in pregnant women vaccinated with Tdap vaccine. Vaccine. 2018;36(34):5173-9.

27. Hall C, Abramovitz LM, Bukowinski AT, Ricker AA, Khodr ZG, Gumbs GR, et al. Safety of tetanus, diphtheria, and acellular pertussis vaccination among pregnant active duty U.S. military women. Vaccine. 2020;38(8):1982-8.

28. Schurink-van 't Klooster TM, De Melker HE. The National Immunization Programme in the Netherlands 2018 - 2019. 2020.

\section{Hosted file}

Table 1. incidence rates per 10,000 of adverse pregnancy and birth outcomes for 2006-2018.docx available at https://authorea.com/users/345862/articles/471959-background-incidence-ratesof-adverse-pregnancy-outcomes-in-the-netherlands-data-of-2006-2018

\section{Hosted file}

Figure 1. Trends per 10,000 of adverse outcomes at maternal level for 2006-2018.docx available at https://authorea.com/users/345862/articles/471959-background-incidence-ratesof-adverse-pregnancy-outcomes-in-the-netherlands-data-of-2006-2018

\section{Hosted file}

Figure 2. Trends per 10,000 of adverse outcomes at neonatal level for 2006-2018..docx available at https://authorea.com/users/345862/articles/471959-background-incidence-ratesof-adverse-pregnancy-outcomes-in-the-netherlands-data-of-2006-2018

\section{Hosted file}

Table 2. incidence rates per 10,000 of adverse outcomes in subgroups of term and preterm infants.docx available at https://authorea.com/users/345862/articles/471959-background-incidence-ratesof-adverse-pregnancy-outcomes-in-the-netherlands-data-of-2006-2018 